\title{
Erratum to: A pragmatic approach to infants with Robin sequence: a retrospective cohort study and presence of a treatment algorithm
}

\author{
Emma C. Paes ${ }^{1,5}$ • Daan P. F. van Nunen ${ }^{1}$ - Lucienne Speleman ${ }^{2}$. \\ Marvick S. M. Muradin ${ }^{3}$ - Bram Smarius ${ }^{1}$ - Moshe Kon ${ }^{1}$. \\ Aebele B. Mink van der Molen ${ }^{1}$ • Titia L. E. M. Niers ${ }^{4}$. \\ Esther S. Veldhoen ${ }^{4} \cdot$ Corstiaan C. Breugem ${ }^{1}$
}

Published online: 9 July 2015

(C) Springer-Verlag Berlin Heidelberg 2015

\section{Erratum to: Clinical Oral Investigations \\ DOI 10.1007/s00784-015-1407-6}

The original version of the above article contained a mistake in the presentation of the name Aebele B. Mink van der Molen. The family name should be Mink van der Molen and not van der Molen alone.

The online version of the original article can be found at http://dx.doi.org/ 10.1007/s00784-015-1407-6.

$\triangle$ Emma C. Paes

emmapaes@gmail.com

1 Department of Plastic, Reconstructive and Hand Surgery,

Wilhelmina Children's Hospital, Utrecht, The Netherlands

2 Department of Otorhinolaryngology, Wilhelmina Children's

Hospital, Utrecht, The Netherlands

3 Department of Oral and Cranio-Maxillofacial Surgery, Wilhelmina Children's Hospital, Utrecht, The Netherlands

4 Department of Pediatrics, Wilhelmina Children's Hospital, Utrecht, The Netherlands

5 Department of Pediatric Plastic Surgery, Wilhelmina Children's Hospital Utrecht, PO Box 85500, 3508 GA Utrecht, The Netherlands 